\title{
Epidemiology in Antarctica?
}

\author{
HILARY KING
}

From the Antarctic Division, Department of Science, Kingston, Tasmania 7150, Australia.

The 20th century has witnessed a growing awareness of epidemiology as the science of public health. It has also seen both an expansion and a shift in emphasis in epidemiological activity. These developments are, in part, a consequence of contemporary changes in the natural history of disease which have their roots in changing social conditions, values, and behaviour; an alteration in the human ecosystem. It is prudent to anticipate further changes, as both a direct and an indirect consequence of technological progress, a dominant characteristic of 20th century society. In their turn, these will lead to new public health needs and problems and thus to new frontiers for epidemiology.

Recently, growing attention has been paid to the opportunities that the polar regions, particularly Antarctica, may offer to an overcrowded and energyrependent world. An increasing number of nations maintain permanent bases in Antarctica, and it is not uncommon for personnel to spend protracted periods of time at such stations. Births, interpersonal unions, and deaths have all occurred in Antarctica in recent times, and both practical and political considerations rule it almost inevitable that human colonisation of the region will continue.

It is from this perspective-the potential for a growing human social system in Antarctica-that the scope for epidemiology to contribute to polar medicine (or, more specifically, to polar public health) will be considered. Features of Antarctic communities that might be exploited in epidemiological investigations of a more general nature will also be discussed. In conclusion, these opportunities will be weighed against the limitations that certain features of Antarctic society will inevitably place upon epidemiological research.

As Antarctica may be unfamiliar and the concept of polar epidemiology surprising to most epidemiologists, a brief description of the physical, historical, and political background to man's presence in Antarctica will be given in order to place past, present, and future activities there in context.

\section{Physical setting}

The Antarctic Circle delineates the northern limit of Antarctica as latitude $66^{\circ} 33^{\prime} \mathrm{S}$. Of more consequence is the Antarctic Convergence, the location in the Southern Ocean where warm surface water originating in subtropical seas meets cold surface water of Antarctic origin, causing an abrupt climatic boundary. South of the convergence, which varies in latitude around the world, lie the Antarctic continent and a number of subantarctic islands. The latter, although well to the north of the Antarctic Circle, nevertheless experience a very harsh climate, and most are exposed to permanent ice and snow. If the average latitude of the Antarctic Convergence is taken to be between $50^{\circ}$ and $55^{\circ} \mathrm{S}$ (ie, of equivalent latitude to Copenhagen or Calgary in the northern hemisphere) it will be appreciated that climatic conditions are more severe in high southern latitudes than in their northern counterparts.

Antarctica is a region of physical extremes, the dominant characteristics being isolation, low temperatures, low humidity, high winds, and, in many places, high altitudes. These features act with negative synergism upon the survival prospects of all forms of life and have proved an effective barrier to all but a few, specialised species. The continent is virtually devoid of plant life. Before the arrival of man, the only successful mammalian colonists were aquatic.

\section{Historical background to man's presence in Antarctica}

The early history of man in the Antarctic is one of remarkable perseverance and hardship. Cook was the first to cross the Antarctic Circle in 1773. The first landing on the continent occurred in 1895 , and the first party to spend the winter there did so in 1898 .

Thus, the 20th century began with the stage set for the exploration and potential exploitation of Antarctica. The experiences of the early explorers drew attention to man's capacity for protracted survival under adverse conditions of the most extreme severity, In the process, many fell victim to diseases directly related to their inadequate resources: hypervitaminosis due to excessive consumption of dog liver, scurvy, carbon monoxide poisoning due to inadequate ventilation in polar tents and huts, frostbite, and snow blindness. Although these conditions are not unique to the Antarctic, they were a major obstacle to the early explorers, ${ }^{1}$ and the latter two still account for avoidable morbidity in Antarctic personnel today.

The International Geophysical Year of 1957-8 provided a major impetus for scientific activity in Antarctica and for the establishment of permanent 
settlements in the region. These settlements, in the form of bases of national designation, are the fulcra of modern human activity in Antarctica. Forty two occupied stations were maintained in the Antarctic by 16 nations during the austral winter of $1985 .^{2}$ The number of both settlements and participating nations continues to grow.

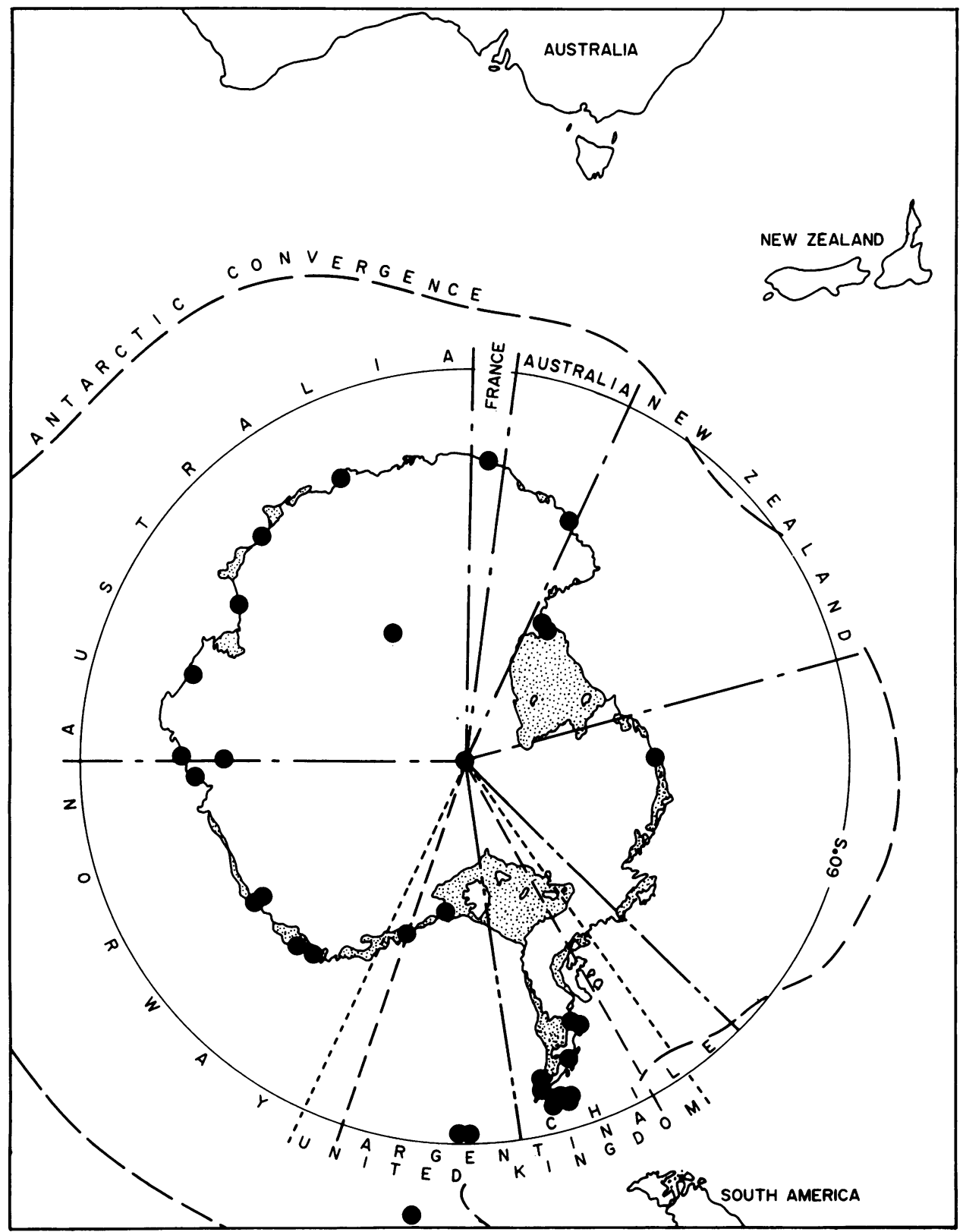

Map of Antarctica, showing the territorial claims of the claimant nations and the location of stations to the south of the Antarctic Convergence which were occupied during the austral winter of 1985.

The residents of Antarctica in winter months (thus excluding the many summer visitors, including tourists, who go there each year) number approximately 1000 at the present time. The fact that such a large population may be found in such an inhospitable location (at no small expense to the 
nations concerned) prompts an enquiry into the motives behind the colonisation of Antarctica.

\section{Political background to man's presence in Antarctica}

From the outset, exploration of the high southern latitudes was economically motivated. By the end of the 19th century, European and American entrepreneurs had established a ring of sealing and whaling installations around the Southern Ocean. Polar exploration also fulfilled an important nationalistic function in Europe at the beginning of the 20 th century, thus earning both popular and political support.

Early in the present century, several nations laid claim to portions of Antarctica (figure). Both the legal basis for these claims and respective territorial boundaries remain open to dispute.

Recently, strategic considerations and the promise of exploitable natural resources-particularly seafood and hydrocarbons - have emerged as powerful incentives for Antarctic activity. Conflicting national aspirations in these areas have the potential to catalyse territorial sentiment and pose a very real threat of international dispute. In an attempt to regulate the activities of interested nations in Antarctica, the Antarctic Treaty was signed in 1959 by the 12 nations involved in the International Geophysical Year Antarctic programme. ${ }^{3}$ The treaty placed a 30 -year moratorium on all territorial claims, prohibited military activity, and encouraged international scientific collaboration in the area south of latitude $60^{\circ} \mathrm{S}$. Scientific cooperation is presently administered by the Scientific Committee on Antarctic Research (SCAR).

A review of the terms of the Antarctic Treaty may be requested by any of the contracting parties after 1991 . Given the potential strategic and economic importance of the region, it may be anticipated with confidence that the nations that have already shown an interest in Antarctic territory will not reduce their physical presence and activity in the region during this critical period. Further colonisation of Antarctica seems almost inevitable; assured in the short term by the proximity of political negotiation and in the long term by strategic considerations and the likely presence of resources which future technological advances may render more readily attainable than they are at present.

\section{Prospects for epidemiology to contribute to polar medicine}

Having established the likelihood of a growing multinational human presence in Antarctica, it is useful to identify epidemiological activities which may be of particular service to medical practice in the polar setting.

HEALTH SURVEILLANCE

A deficiency which becomes immediately apparent when attempting to gather information on health and disease in Antarctica is the lack of a standardised, longitudinal system for recording health-related events. Assisted by recent improvements in new technology such registers are playing an increasingly important role in epidemiology for routine monitoring of a wide range of conditions and for health surveillance of whole populations. They also constitute a valuable resource for generating and testing hypotheses and, additionally, may provide a baseline for the evaluation of intervention measures.

Small, retrospective health surveys have been conducted by motivated Antarctic medical officers, and the proceedings of an international symposium on human biology in Antarctica appeared over a decade ago. ${ }^{4} \mathrm{~A}$ first step in polar health surveillance was laid by the decision by SCAR to develop a standardised procedure for recording accidental injuries in Antarctica. ${ }^{5}$ A United States committee on polar biomedical research recently concluded that the highest priority at the present time is the establishment of a comprehensive registry of health events in polar communities. ${ }^{6}$

In the confines of an Antarctic base, ascertainment of overt ill health should be complete, and simple standardised procedures could enable the accumulation of accurate health statistics on a routine basis. Such a data base could be used to identify key problems in polar public health, to monitor secular trends, and eventually to assess preventive strategies. The value of a system of this sort would be greatly enhanced if it could be developed on a multinational basis, with the establishment of an international coordinating centre for health surveillance in Antarctica. The centre should have the facilities to store, retrieve, and analyse epidemiological data and to disseminate the information routinely, in the form of regular reports similar to those produced by the international health agencies from other parts of the world. Such surveillance would represent the first step in coordinated epidemiological activity in Antarctica.

\section{PRIMARY PREVENTION}

Since most personnel serving for any length of time in Antarctica are subjected to a screening health examination, the population could be expected to be healthy. It is not surprising therefore that the majority of deaths and serious medical problems appear to be due to accidental injury. High latitudes appear to be 
associated with high morbidity and mortality from accidents in both hemispheres; the accident mortality rate in Alaska is almost twice that for the United States as a whole. ${ }^{7}$ Insomuch as each of these events is potentially avoidable, their occurrence warrants careful study in an attempt to maximise safety in the Antarctic workplace. Serious illness in the remote location of Antarctica is not only a personal disaster, it also constitutes adverse publicity for polar activities and frequently leads to very costly, time consuming, and hazardous evacuation. ${ }^{8}$

A procedure for recording accidents in Antarctica was introduced by SCAR a decade ago (Report of the Working Group on Human Biology and Medicine to the 14th meeting of SCAR, Mendoza, 1976) but results from this project have yet to be published. Data relating to all accidents on Australian National Antarctic Research Expeditions during the years 1977-85 have recently been collated and were presented at the most recent meeting of SCAR in San Diego in June 1986 (D J Lugg, personal communication). It is to be hoped that this will stimulate the production and dissemination of similar material from other Antarctic communities.

In addition to quantifying and describing the nature of accidental injuries and determining the circumstances in which they occur, it may be possible to assess the characteristics of subjects prone to accident in the Antarctic environment. This would offer the opportunity for improved screening of prospective recruits and also for enlightened modification of training programmes.

Hypothermia, frostbite, and snow blindness are avoidable illnesses resulting from inadequate protection from the polar environment, and they should be considered in the same light as accidental injury. The occurrence of these conditions should be carefully monitored. International collaboration on the collation and analysis of information on accidents and avoidable illness in Antarctica may be a first step towards their control.

\section{SOCIAL MEDICINE}

Psychological problems have been associated with Antarctic residence since the earliest days of exploration. $^{1}$ A feature of life in small, isolated communities is the need for prolonged co-existence with a small number of colleagues on very close quarters. Abrogation of personal privacy and restricted social intercourse may prove very stressful for individuals unaccustomed to such constraints. Furthermore, limited recreational facilities and an inclement environment may exacerbate the problem.

These aspects of the Antarctic microcosm offer challenging opportunities for study in the elusive field of human social adjustment and coping mechanisms, To date, investigation has included the development of performance criteria, validation of psychological screening of recruits, and the study of attitude changes during residence in Antarctica. ${ }^{9}$ Adaptation of a similar nature is required of subjects in other circumscribed environments, and knowledge gained in Antarctica could well be applied elsewhere.

No information exists on delayed physical and psychological consequences of temporary residence in polar regions. As the number of subjects exposed to this experience grows, the notion that it may result in adverse influences on physical and mental health, which are not immediately apparent, can no longer be ignored. A longitudinal study of subsequent mortality and morbidity in former polar expeditioners is required.

\section{Prospects for polar medicine to contribute to} epidemiology

In addition to identifying the areas where the $\stackrel{0}{+}$ epidemiological approach may be of value to polar medicine, it is of interest to consider special attributes-perhaps overlooked hitherto-that the unusual Antarctic communities and the environment may offer to epidemiologists not specifically concerned with polar medicine.

\section{POPULATION ISOLATES}

It has been recognised that small population isolates can offer special opportunities for medical research, particularly in the study of infectious disease. Until recently, there were many communities whose contact with the outside world was so sporadic that the epidemic nature of infectious disease could be studied there in a virtual laboratory setting; however, the global mass transit system bypasses very few of these communities today. The majority of Antarctic settlements remain completely isolated from the outside world for a substantial proportion of the year, representing a rare resource for the study of immune mechanisms and the natural history of ubiquitous infectious diseases.

\section{A GROWING DATABASE FOR PROSPECTIVE STUDY}

Most Antarctic personnel submit to extensive medical examination before appointment. Information recorded on all prospective Australian Antarctic expeditioners includes personal and family histories of past illness, full physical examination (including anthropometric indices and blood pressure), chest $x$-ray, electrocardiogram, blood grouping, and certain 
viral titres. Sophisticated storage and retrieval systems are facilitating the collection and use of such material which, routinely gathered during this medical screening, would otherwise entail considerable expense to an investigator.

This information could provide a valuable baseline for longitudinal study of the subsequent development of chronic disease and other delayed conditions. A precedent exists for research of this nature in the longitudinal studies of selected cohorts such as college graduates, public servants, and industrial workers. Such investigation could be designed so as to complement the study of delayed effects of Antarctic residence on the health of expeditioners.

HEALTH IN ARTIFICIAL ENVIRONMENTS OF THE FUTURE

Technological advances are not only altering existing societies, they are also leading to the creation of new ones. By enabling increasing numbers of people to live in increasingly unnatural conditions for an increasing length of time, such advances may create a milieu suitable for long term human habitation in a region that was hitherto the preserve of the pioneer and the explorer. Like any naturally inhabited environment, such a microcosm will contain a population at risk of ill health, and therefore one of relevance to the epidemiologist.

This premiss has an important bearing on possible future directions in epidemiology. Research in Antarctica has the opportunity to make a useful contribution to the study of human health in a new generation of artificial and constrained environments. Examples that come immediately to mind are the communities living on offshore oil installations and future settlements in outer space. Offshore health is already a recognised discipline. ${ }^{1011}$ The study of human health in outer space may represent the final epidemiological frontier. Recent reports of the life science experiments aboard Spacelab 1 raise the possibility that such a development is not far away.

\section{Limitations for epidemiology in polar regions}

It must be acknowledged that there will always be severe limitations to the epidemiological study of small, unrepresentative groups of subjects. Three key issues are those of small sample size, non-random selection, and the artificiality of the environment.

Small sample size can, to some extent, be overcome by studies incorporating several communities, thus providing further impetus for international collaboration in polar medicine. Bias relating to selection and environment will place severe, if not insuperable constraint upon extrapolation of findings from some Antarctic research. However, such studies remain pertinent within the polar context, and some will also be relevant to a growing number of similarly artificial environments. It is important to realise that however unrepresentative Antarctic communities may be of human society as a whole, they nevertheless represent defined populations in their own right, thus fulfilling the cardinal criterion for epidemiological investigation.

\section{Conclusion}

It seems almost certain that the multinational human presence in Antarctica will continue to grow. To date, little attention has been paid to the opportunites for epidemiology to contribute to polar medicine on the one hand, and for the unusual Antarctic communities to be espoused by epidemiologists for the special merits that they may posess on the other.

On the negative side, a considerable proportion of conventional epidemiological research, which relies upon relatively large, naturally occurring populations, could not be carried out in the present polar setting. To this extent, polar epidemiology may be greeted with scepticism by many traditional epidemiologists.

On the positive side, a clear need exists for an international registry system for the routine recording and dissemination of polar health statistics. The study of accidents and avoidable injury, psychological aspects of living in socially constrained conditions, and the long-term consequences of residence in Antarctica are specific areas that require investigation using standard epidemiological methods. Antarctic personnel also offer certain opportunities for epidemiological studies of a more general nature, particularly those relating to population isolates and prospective studies relying on a large, routinely collected database.

All of these activities should be considered in the light of a potential expansion of epidemiological research to other artificial communities and eventually to human adaptability to the most extreme conditions of outer space. Within this context, Antarctica may point the way to a new direction for epidemiologypresently limited in scope, but with growing application in an increasingly technological world.

I am grateful to Dr D Lugg and Dr P Gormly for advice, and to Mr B Hill for the preparation of the figure.

\section{References}

${ }^{1}$ Lugg D J. Antarctic medicine, 1775-1975. 1. Med J Aust 1975(2): 295-8. 
2 SCAR Bulletin No. 81. Stations operating in the Antarctic, winter 1985. Polar Record 1985; 22(141): 733.

3 The Antarctic Treaty. Miscellaneous No. 21(1959). Cmnd. 913, London, H M Stationery Office, 1959.

${ }^{4}$ Edholm O G, Gunderson E K E (eds). Polar human biology. Proceedings of the SCAR/IUPS/IUBS Symposium on Human Biology and Medicine in the Antarctic. London; Heinemann, 1973.

5 SCAR Bulletin No. 49. Thirteenth Meeting of SCAR, Jacksons Hole, 3-7 September 1974. Appendix C. Working Group on human Biology and Medicine. Polar Record 1975; 17(109): 446.

6 Ad Hoc Committee on Polar Biomedical Research. Polar biomedical research: an assessment. Washington: National Academy Press, 1982, 11-15.
${ }^{7}$ Northern Alaska Health Resources Association. Accident prevention plan 1981. Fairbanks, 1981.

${ }^{8}$ Podkolinski M T, Semmens K. Intestinal haemorrhage in Antarctica, a multinational rescue operation. Med J Aust 1979 (2): 275-7.

${ }^{9}$ Gunderson E K E. Psychological studies in Antarctia: a review. In: Edholm O G, Gunderson E K E (eds). Polar human biology. Proceedings of the SCAR/IUPS/IUBS Symposium on Human Biology and Medicine in the Antarctic. London, Heinemann, 1973, 352-61.

${ }^{10}$ Cox RAF (ed). Offshore medicine. New York: SpringerVerlag, 1982.

11 Norman J N, Brebner J. The offshore health handbook. London: Dunitz, 1985.

12 Abelson P H. Spacelab 1. Editorial. Science 1984; 225: 127. 